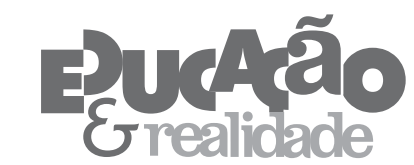

\title{
Territorialidad y Neoliberalismo: el caso de la Universidad Estatal de Aysén en Chile
}

\author{
Francisco Durán del Fierro' \\ Roxana Pey Tumanoff"
}

'University College London (UCL), London - Reino Unido

"Universidad de Chile, Santiago - Chile

RESUMEN - Territorialidad y Neoliberalismo: el caso de la Universidad Estatal de Aysén en Chile. Este artículo reflexiona sobre la experiencia de la Universidad de Aysén como subversidad. Para ello, analizamos cómo la Universidad ha traducido las políticas nacionales y las expectativas de la comunidad local en prácticas institucionales orientadas a concretar el proyecto territorial. Así, en primer lugar, exploramos la imposibilidad de la Universidad de convertirse en subversidad en función de las tensiones que derivan de las políticas de mercado. En segundo lugar, indagamos en la posibilidad de la subversidad a partir de la constitución del Consejo Social. Este análisis nos permite concluir que la Universidad contiene una promesa que descansa en la capacidad del Consejo Social de repensar los principios fundantes de la Universidad.

Palabras-clave: Subversidad. Universidad Territorial. Participación Social. Políticas de Mercado.

ABSTRACT - Territoriality and Neoliberalism: the case of the Universidad Estatal de Aysén in Chile. This article reflects on the experience of the Universidad de Aysén as subversity. In order to do that we analyze how the University has translated national policies and local community's expectations into institutional practices aimed at materializing the territorial project. First, we explore the impossibility of the University become subversity in light of the tensions ensuing from market-oriented policies. Second, we delve into the possibility of the subversity through the analysis of the Social Council. This analysis leads us to conclude that the University has a promise that rests upon the Social Council's capacity of rethinking the foundational principles of the University.

Keywords: Subversity. Territorial University. Social Participation. MarketOriented Policies.

Educação \& Realidade, Porto Alegre, v. 46, n. 4, e118081, 2021. 
Territorialidad y Neoliberalismo

\section{Introducción: la constitución de un proyecto territorial}

Las universidades son un espacio social orientado a la producción y diseminación del conocimiento, además de promover el desarrollo social, cultural y económico de los países y sus territorios (Marginson, 2011). Ese doble carácter ha estado inscrito en la historia de las universidades latinoamericanas, especialmente en Chile, desde la gestación de la primera universidad en el siglo XIX (Bernasconi, 2008). Sin embargo, la modernización de la educación superior desde la década de los noventa en adelante, principalmente por medio de políticas de mercado, ha reinterpretado dichos principios fundantes, o más bien, los ha vaciado de toda referencia vinculada al desarrollo nacional (Villalobos-Ruminott, 2018), que sumado a la desarticulación de los sistemas educativos y las resistencias conservadoras ha llevado a la crisis de la Universidad (Pey, 2006). En 2011 y 2012, dicho proceso modernizador fue impugnado por la sociedad chilena. El rol del Estado y el mercado fueron profusa y agudamente problematizados, y los gobiernos se vieron obligados a repensar las políticas e instrumentos utilizados hasta ese momento. En ese contexto, de diversas impugnaciones y tensiones, de álgido debate nacional, de controversias públicas, es posible entender el sentido de la Universidad Estatal de Aysén, de una universidad pública que nace como una alternativa plausible al modelo privado y neoliberal de universidades, desde un territorio aislado y extremo (Pey, 2018).

A diferencia de otras universidades estatales y privadas, las que nacieron por decisiones administrativas, inversiones de grupos empresariales, o de grupos de élite dentro de comunidades locales (Brunner, 2011), la Universidad de Aysén es la primera institución de educación superior en Chile que se crea exclusivamente como consecuencia de un movimiento social ${ }^{1}$. Los problemas derivados de la persistente desigualdad social y territorial de la región explican en gran medida la fuerza de la movilización, la que se prolongó por meses y resonó en todo el país por su alcance, profundidad y exigencias por mayor justicia social (Pérez, 2015). Asimismo, los problemas y deficiencias del sistema de educación superior, particularmente vinculados con los mecanismos regulatorios y de financiamiento, además de la equidad y calidad, fueron el fundamento de las propuestas del movimiento social en materia de educación superior. A diferencia de otras experiencias, como las universidades populares en América Latina, Europa o el norte de África ${ }^{2}$, la Universidad de Aysén es creada por el Estado. Sin embargo, en el caso de Aysén esto no necesariamente debe considerarse como una carencia de activismo o dependencia estatal. Al contrario, antes de la creación de la Universidad la región ya había definido sus principios orientadores a través de un proceso participativo que condujo a lo que se denominó "otro modo de habitar el planeta" (Comisión Ejecutiva Regional, 2015).

Dado que la universidad nace como una demanda de la región es que se vuelve relevante indagar cómo la institución se vincula con la comunidad local. Dicha relación es crucial puesto que es la forma en que el espíritu del proyecto transformador persiste en la universidad. Por tal motivo, es importante hablar de universidad territorial en tan- 
to el adjetivo territorial indica su carácter fundante, pero también su sentido de ser más profundo, como lo fue en su momento el proyecto de universidad nacional en los albores de la República. Además, el hecho de ser una universidad territorial contiene una impugnación y una promesa. Es la impugnación a las políticas de mercado que no diferencian territorios y localidades, que no valoran la diversidad de necesidades sociales, y que toman decisiones centralizadas basadas en abstracciones cuantitativas (Villalobos-Ruminott, 2018). De cierta manera, es la impugnación a la universidad emprendedora y a la universidad docente dominante en el proyecto modernizador Chileno (Rivera-Polo et al., 2020). En otras palabras, es la interpelación a un proyecto modernizador basado en una perspectiva capitalista y colonialista (Santos, 2018). Por otra parte, es una promesa en tanto que busca reactivar los principios de conocimiento y desarrollo en un territorio aislado y extremo. Es la promesa de una universidad que valora a su comunidad circundante como principal fuente de valor y conocimiento, y que contribuye a su desarrollo social, cultural y económico. En definitiva, es la promesa de una universidad que transforma el territorio y que se transforma por el territorio (Comisión Ejecutiva Regional, 2015).

En ese contexto, este artículo interroga la existencia de la Universidad en tanto universidad (im)posible (Thayer et al., 2018) y subversiva (Santos, 2018) en un sistema altamente mercantilizado. En particular, nos interesa reflexionar sobre los mecanismos que permiten constituir la idea de universidad territorial en la región de Aysén, especialmente en aquellos que la definen estatutariamente como lo es el Consejo Social (CS). A partir de ello, el artículo busca comprender cómo la Universidad ha enfrentado, traducido y articulado las expectativas del movimiento social de Aysén, las emergentes necesidades de la región y el cumplimiento de las políticas nacionales. En particular, nos interesa explorar las tensiones y desafíos que genera el CS, como elemento formal consignado en su Estatuto, en la propia universidad, y elemento distintivo dado que no hay otra universidad en el país que incluya un consejo con estas características.

El artículo se divide en tres secciones. La primera sección describe el contexto y las tensiones que dieron origen a la Universidad. En segundo lugar, se discute la idea de universidad territorial como subversidad para comprender el carácter transformador e impugnador de la Universidad de Aysén respecto de las reglas y políticas que le dieron origen. En tercer lugar, se analizan las diversas respuestas de la universidad frente a las políticas del gobierno central (artículos transitorios de la ley 20.842) y regional, y las expectativas de la comunidad local. Al respecto, se explora en unas de las medidas más relevantes de la universidad como respuesta a estas políticas e interacciones: el Consejo Social. Así, se analiza en qué medida el CS es el elemento que permite pensar la universidad de Aysén como subversidad. Para ello, se discute cómo el CS impugna a la propia universidad. Este análisis proporciona evidencia sobre las perspectivas, desafíos y tensiones que enfrenta un proyecto de universidad territorial frente a un sistema universitario altamente mercantilizado.

Educação \& Realidade, Porto Alegre, v. 46, n. 4, e118081, 2021. 
Territorialidad y Neoliberalismo

\section{La Universidad Estatal de Aysén: territorial, extrema y subversiva}

\section{Movimiento Social de Aysén y la Reforma a la Educación Superior}

El desarrollo de una universidad territorial depende en gran medida de las condiciones institucionales y de financiamiento del sistema de educación superior. En Chile, dichas condiciones han limitado la agencia de las universidades territoriales, en particular de aquellas que se ubican en las zonas más extremas del país. La consolidación de la agenda neoliberal desde la década de los noventa en adelante (Salazar Zegers; Leihy, 2013) ha producido un sistema altamente diversificado y diferenciado, en todos sus niveles (Lemaitre, 2019; Van Vught, 2009). Sin embargo, se trata de una diversidad guiada por criterios puramente técnicos que no distinguen entre territorios y localidades, transformando a la pretendida diversidad en un riesgo para el sistema (Lemaitre; Durán del Fierro, 2013). Se trata de una diversidad que, paradójicamente, premia ciertas formas de universidad mientras castiga otras. Las universidades territoriales han sido justamente aquellas más afectadas por dichas definiciones.

El modelo de educación superior en Chile incentiva la competencia por estudiantes, fondos de investigación y académicos (Ossa, 2016). En estas condiciones, las universidades regionales han tenido que tomar decisiones drásticas para sobrevivir, como la apertura de sedes y carreras en zonas con mayor población, con el objetivo de obtener recursos a través de la expansión de la matrícula (competencia cuantitativa), debilitando su proyecto territorial. Esto ha producido la concentración de la matrícula en la Región Metropolitana, la que en 2020 alcanzó el 45,7\% de la matrícula total (SIES, 2020). Además, las universidades regionales han tenido que modificar aspectos centrales de su gobernanza y gestión para adecuarse a las exigencias de la acreditación institucional y de carreras, es decir, ajustarse a la competencia cualitativa (Musselin, 2018), lo cual ha reducido la diversidad de proyectos y homogeneizado las formas de organización institucional.

Es importante mencionar que la región de Aysén fue el único territorio nacional que, después de la expansión de la educación superior a través de colegios universitarios de la Universidad de Chile en la década del 60 y 70, no contaba con una Institución de Educación Superior (IES). Luego, a través de distintos procesos, tanto universidades estatales como privadas crearon sedes regionales con el objetivo de ofrecer mayores oportunidades de estudio a los jóvenes y adultos de la región (Comisión Ejecutiva Regional, 2015). Sin embargo, las persistentes desigualdades educativas en términos de logro académico junto con las escasas oportunidades que brindaban las instituciones en esa época, cuyo vínculo territorial descansaba simplemente en la docencia de pregrado, contribuyeron a darle sentido a la demanda de una universidad pública en Aysén (Pérez, 2015). Adicionalmente, es relevante señalar que la región enfrentaba - y lo sigue haciendo - desafíos en energía, sa- 
lud, medio ambiente, conectividad, pobreza, desigualdad, relación con pueblos originarios, entre otros (Comisión Ejecutiva Regional, 2015).

La fuerza del movimiento social se explica, en parte, por la historia de la región. Los primeros habitantes se asentaron luego de varias exploraciones a la Patagonia. A comienzos del siglo XX, los primeros colonos, dado el aislamiento de la región, decidieron fundar pueblos, abrir rutas, y explotar la tierra por su propia iniciativa. El Estado llegó mucho después solo para regularizar aspectos legales y técnicos. En otras palabras, se trata de un territorio que se ha constituido sin la ayuda del Estado, lo que explica la identidad y autonomía territorial (Comisión Ejecutiva Regional, 2015). Esta situación de aislamiento, precariedad y desamparo ha caracterizado la historia social de la región, y fue lo que de alguna manera detonó la revuelta del 2011 y las demandas que de ahí nacieron.

Durante 2011 y 2012 las protestas se intensificaron en la región a tal punto que el gobierno central tuvo que negociar una salida política y acordar un conjunto de soluciones. La creación de una universidad estuvo entre los puntos centrales. Sin embargo, el primer gobierno de Sebastián Piñera rechazó la idea de una universidad pública en la región. Los motivos esgrimidos fueron principalmente económicos: no hay suficiente demanda para aumentar la oferta educativa en la región, es decir, el tamaño del mercado no es suficiente para estimular la competencia por calidad (Rivera-Polo et al., 2018). Es el segundo gobierno de Michelle Bachelet el que asume el compromiso de crear la universidad en la región, como parte de la Reforma a la Educación Superior (20162018). En el contexto de esta reforma, el programa de gobierno propuso la creación de dos nuevas universidades estatales, una en la región de O'Higgins y otra en la región de Aysén. La decisión de crear la primera fue meramente administrativa, bajo el principio de igualdad que contiene la constitución y que impide decisiones diferenciadas aunque estas sean necesarias. La razón de crear la segunda, como hemos visto, provino de la exigencia del Movimiento Social de Aysén. A pesar de estas diferencias, una ley común crea ambas universidades. Así, en 2015, el gobierno promulga la ley 20.842 que crea las dos nuevas instituciones, estableciendo, entre otras cosas, los plazos de su instalación, los criterios para elegir a sus primeras autoridades y la forma y condiciones generales para definir los estatutos.

\section{La universidad territorial como subversidad}

Es posible sostener que existe una tensión entre la agenda neoliberal y la idea de universidad territorial. Como señala Rivera-Polo (2018), una universidad territorializada tiene particularidades distinguibles del resto de las universidades. Así, una universidad territorial se desarrolla principalmente en función de las necesidades del territorio, y no como resultado de la relación oferta y demanda. Adicionalmente, la universidad territorial no puede ser meramente docente. El impulso y consolidación del desarrollo social, cultural y económico de una re- 
gión o localidad descansa en la interrelación entre docencia e investigación. Finalmente, la universidad territorial es pensable en la medida que reduce las barreras de entrada, principalmente económicas, a los estudiantes y requiere de un cuerpo académico comprometido y heterogéneo. Por lo tanto, es posible sostener que las universidades territoriales son importantes puesto que impulsan el desarrollo integral en las localidades en que se ubican (Rock et al., 2013).

Teniendo en cuenta estas consideraciones, la universidad territorial puede concebirse como subversidad en los términos propuestos por Santos (2018). Según el autor, la subversidad es distinguible en los siguientes ámbitos. En primer lugar, su audiencia es amplia y heterogénea, es decir, busca incluir a todos los actores sociales que han sido excluidos socialmente y que han sufrido injusticias sistemáticas derivadas de la pertenencia a una clase social, género, etnia, religión, etcétera. En segundo lugar, promueve una pedagogía que reconoce una diversidad de fuentes de saber, es decir, configura espacios de aprendizaje donde se valoran los conocimientos artesanales y se crean comunidades que construyen su propio conocimiento. En tercer lugar, proporciona un ambiente de aprendizaje y estrategia pedagógica estrechamente conectada con las luchas sociales. De esa manera, contempla una pedagogía pragmática o del conflicto que busca fortalecer la lucha social contra todo tipo de exclusión y discriminación y, a la vez, promover el diálogo entre grupos que concurren con diferentes perspectivas o universos simbólicos. Por último, la subversidad puede tener su origen en movimientos sociales y, por tal motivo, se ubica en una variedad de lugares, tales como valles remotos, montañas, prisiones, etcétera. Asimismo, los académicos de la subversidad, en vez de buscar la promoción académica, están comprometidos con la lucha social y abiertos a volver a aprender (Santos, 2006; 2018).

La Universidad de Aysén, en algún sentido, puede ser pensada como subversidad, particularmente en relación con su origen (movimiento social), ubicación (localidad aislada y extrema) y contexto (modelo chileno de educación superior intensamente neoliberal). Sin embargo, la capacidad de convertirse en subversidad radica en que los dos primeros elementos puedan presionar de manera permanente el funcionamiento interno de la universidad, es decir, al gobierno y gestión universitaria, la docencia, la investigación y el rol de sus autoridades y cuerpo académico, y que el tercero, el modelo de educación superior prevalente, esté en retirada, como aparentemente lo estaba cuando se creó la universidad. Por lo tanto, es posible sostener que ambos elementos - energía fundante y localidad - pueden modificar a la Universidad y convertirla plenamente en subversidad. Lo crucial es cómo dichos elementos son canalizados y articulados por la propia universidad, cambiándola y, a su vez, cambiando al territorio. Como el mismo Santos (2018) lo advierte, la configuración de la subversidad queda supeditada a dos factores que convergen. Por una parte, las expectativas del movimiento social y de los grupos tradicionalmente excluidos de la educación superior. Y, por otra parte, el ajuste de la universidad a las políticas nacio- 
nales y globales. El análisis de dicho vínculo y tensiones es el propósito de este artículo, es decir, explorar en qué medida los mecanismos de vinculación de la universidad permiten reconocer y articular las necesidades del territorio y, por tanto, transformarlo y, a su vez, cómo a través de esa articulación la propia universidad es tensionada y transformada.

\section{Metodología}

Este artículo tiene como objetivo principal analizar cómo la Universidad Estatal de Aysén ha traducido las políticas nacionales y regionales, y las tensiones que emergen entre las expectativas de la comunidad local y las prácticas institucionales. En este caso, se analiza cómo la universidad ha traducido las exigencias contenidas en las normativas de la ley que la crea (ley 20.842) y las políticas nacionales. En particular, el artículo se enfoca en analizar la respuesta más innovadora de la universidad en dicho contexto de políticas y expectativas: el Consejo Social. Así, busca comprender la relación entre las políticas y las respuestas institucionales (Ball et al., 2012). Dado que las políticas establecen orientaciones generales, las instituciones tienen espacio para responder y traducir dichas exigencias a través de una variedad de prácticas. Por esa razón, si bien la ley 20.842 crea la Universidad de O'Higgins y la Universidad de Aysén bajo las mismas reglas, ambas han traducido dichas prerrogativas de distinta manera.

El estudio asume una aproximación cualitativa para responder al objetivo de investigación. Como señala la literatura en este ámbito, los estudios de casos no buscan representatividad sino más bien profundidad y comprensión del objeto de estudio con miras a explorar las características significativas de los casos (Bassey, 1999; Yin, 2003). En este sentido, la Universidad de Aysén ha sido seleccionada como un caso relevante puesto que ha sido recientemente creada como resultado de las demandas sociales de la zona, las que impugnaron el poder político de la época y a las políticas de mercado implementadas durante las últimas décadas.

Para la producción de información, se realizaron dos procesos en paralelo. En primer lugar, un análisis de los principales documentos elaborados a partir de la creación de la Universidad. El objetivo de este análisis fue indagar en los distintos conflictos y tensiones que subyacen al proyecto de la Universidad de Aysén. En segundo lugar, se realizaron entrevistas en profundidad y semi-estructuradas a distintas personas vinculadas a la universidad desde su creación hasta la actualidad. En ambos casos, los datos fueron analizados a través de la técnica denominada análisis temático (Joffe, 2011). El siguiente cuadro resume ambos niveles de producción empírica. 
Territorialidad y Neoliberalismo

Tabla 1 - Muestra de documentos analizados y perfil de personas entrevistadas

\begin{tabular}{|c|c|}
\hline Documentos analizados & Personas entrevistadas \\
\hline 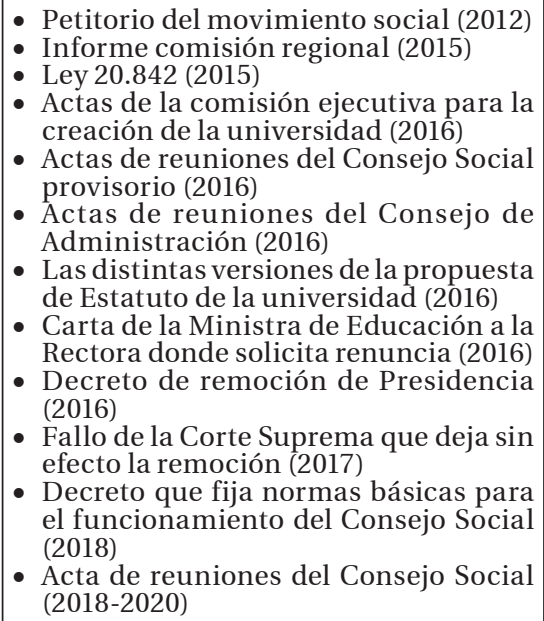 & $\begin{array}{l}\text { - Ex autoridades de la universidad (2015- } \\
\text { - Auto } \\
\text { 2021) } \\
\text { - Miembros de la comisión regional de la } \\
\text { universidad que surgió del movimiento } \\
\text { - social (2015) } \\
\text { Miembros delConsejo Social provisorio } \\
\text { (2016) } \\
\text { Miembros del actual Consejo Social } \\
\text { (2021) }\end{array}$ \\
\hline
\end{tabular}

Fuente: Elaboración propia.

\section{La (im)posibilidad de la subversidad: el devenir de la Universidad Estatal de Aysén en Chile}

A partir de los datos analizados, dos temas nos permiten comprender el devenir de la universidad en relación con el marco teórico propuesto. Por una parte, la imposibilidad de la Universidad de Aysén de convertirse en 'subversidad'. Y, por otra parte, la Universidad contiene una posibilidad, aquella de convertirse en 'subversidad' a partir de la existencia del Consejo Social (CS).

\section{La imposibilidad: la Universidad de Aysén en el modelo neoliberal}

La Universidad de Aysén, si bien emerge como consecuencia del movimiento social de Aysén, es, en la práctica, una universidad estatal. Esto implica que debe cumplir con las normativas de la ley que la creó y con las políticas nacionales vigentes. La Universidad fue creada en 2015 a través de la ley 20.842, la que estableció la forma y condiciones para su instalación. Así, por ejemplo, la ley definió que el rector/a de la universidad debía presentar la propuesta de estatutos al Ministerio de Educación y al Ministerio de Hacienda durante el primer año de funcionamiento. Además, la ley estableció que la universidad debía ser tutelada por otra universidad con el propósito de garantizar su calidad (Chile, 2015). Así, el primer año estuvo dirigido a elaborar los estatutos de la universidad, definir la infraestructura, la política académica, de inves- 
tigación y de aseguramiento de la calidad, junto con crear las primeras carreras y programas.

A partir de los documentos analizados durante el período de instalación (2015-2016), tres elementos muestran las tensiones entre la universidad y el modelo de educación superior imperante en Chile: 1) el sistema de financiamiento; 2) el sistema de admisión; y 3) el gobierno universitario ${ }^{3}$.

Financiamiento: igualdad versus focalización - La Reforma a la Educación Superior (2014-2018) contempló la introducción de una política de gratuidad para los estudios de pregrado. La medida incluyó un conjunto de condiciones para los estudiantes e instituciones. Así, para acceder a los beneficios los estudiantes deben cumplir con un conjunto de requisitos, entre los cuales destaca la condición socioeconómica. Por su parte, las instituciones deben estar acreditadas, participar del Sistema Único de Acceso (SUA) y disponer de políticas que permitan el ingreso de estudiantes de diversos estratos socioeconómicos ${ }^{4}$.

En ese contexto, durante la instalación de la universidad, las autoridades propusieron un modelo de financiamiento estudiantil distinto al planteado por el gobierno, es decir, uno de carácter universal ${ }^{5}$. Así quedó constatado en la segunda acta del Consejo Social provisorio, donde se señala que "[...] se está planteando que el alcance de la gratuidad para esta universidad no se limite al porcentaje de los menores quintiles como se está haciendo a nivel país, sino que acá se otorgue la gratuidad total" (Universidad de Aysén, 2016a, p. 1). Los argumentos esgrimidos por las autoridades de la época tuvieron especial consideración el contexto regional en la medida que la mayor parte de los estudiantes que se quedan en la región después de terminar la educación secundaria provienen de familias de escasos recursos. Además, las proyecciones realizadas por la universidad indicaban que el tamaño de la primera cohorte sería pequeño, de alrededor 300 estudiantes, motivo por el cual el financiamiento basal - asegurado por la ley que la creo - permitía su funcionamiento sin necesidad de ingresos adicionales vía cobro de aranceles (Universidad de Aysén, 2016a). En otras palabras, la Universidad de Aysén nació con un presupuesto basal, que es justamente la forma de financiamiento institucional rechazada por el modelo imperante en Chile.

Admisión: inclusión versus meritocracia - En Chile, todas las universidades estatales pertenecen al SUA. Esto implica que la Universidad debe cumplir con las directrices y criterios definidos por el sistema, dejando escasa flexibilidad para modificar sus mecanismos. El principal instrumento utilizado para seleccionar a los estudiantes es la Prueba de Selección Universitaria (PSU) ${ }^{6}$, la que, en simple, evalúa los contenidos curriculares de la educación secundaria. Sin embargo, las cifras en esos años (rendimiento académico de los estudiantes) no eran muy alentadoras para las autoridades de la Universidad. Por ejemplo, solo la mitad de los estudiantes que egresaron de la educación secundaria en 2016 rindieron la PSU, y de los que la rindieron, un $49 \%$ de ellos obtuvo un puntaje promedio bajo los 450 puntos. De hecho, entre 2013 y 2016 
Territorialidad y Neoliberalismo

el puntaje promedio en la región fue de 383 puntos (Durán del Fierro, 2016).

Frente a este panorama, la Universidad decidió que era urgente y necesario repensar algunos aspectos del sistema de acceso en la región, sin necesariamente eliminar la idea de mérito que lo justifica. Así, por ejemplo, en la segunda sesión del Consejo Social provisorio, el día 01 de Julio de 2016, la Rectora señaló que

\begin{abstract}
[...] estamos trabajando en establecer un mecanismo regular, inclusivo, para hacernos cargo de la realidad que debemos atender acá en la Región, ya que vamos a seleccionar a los mejores estudiantes, pero no entendemos por mejores a los más altos puntajes PSU, sino los que en sus colegios, comparativamente, son los mejores, aunque en ese colegio no haya recibido todas las materias (Universidad de Aysén, 2016a, p. 3).
\end{abstract}

Debe considerarse que la ley 20.842 de creación de la Universidad, establece que se debe "[...] propender a la incorporación de estudiantes provenientes de las regiones respectivas considerando las necesidades específicas de cada zona, a través, por ejemplo, de programas de ingreso especial" (Chile, 2015, p. 4). Las autoridades de la época asumieron este mandato con el conocimiento de que era prácticamente imposible matricular estudiantes con los criterios usados por el resto de las universidades del país. Así quedó definido en la tercera sesión del Consejo Social provisorio, el día 21 de Julio del 2016, donde se acordó que

Se enviaría carta al Consejo de Rectores de las Universidades Chilenas solicitando acoja la petición de un sistema especial de ingreso a la Universidad de Aysén, de acuerdo a propuesta que ya fue presentada por la Rectora, que considere una bonificación a los estudiantes de la Región de Aysén (Universidad de Aysén, 2016b, p. 4).

En concreto, la propuesta buscaba disminuir las barreras de acceso a través de la bonificación de un $20 \%$ al puntaje promedio de la PSU para los estudiantes de la región que quisieran estudiar en la Universidad. Asimismo, se propuso modificar el peso de las ponderaciones del sistema de acceso, dándole mayor importancia al rendimiento académico en la educación secundaria que a la prueba estandarizada ${ }^{7}$.

Gobierno universitario: democracia versus eficiencia - Sumado a las tensiones sobre financiamiento y admisión que generaron durante el primer año de existencia las propuestas provenientes de las autoridades de la universidad, debe agregarse que el texto del Estatuto también produjo contrariedad en el gobierno central, a tal punto que se pidió la renuncia de la Rectora y se inició un proceso de remoción. Ese proceso evidencia la imposibilidad de subversidad dentro del modelo neoliberal imperante contra el que se enfrentaba esta institución naciente para cumplir el mandato social de territorialidad y para cumplir la propia ley que le daba origen. La carta enviada por la Ministra de Educación de la época a la Rectora de la universidad es clara al respecto. En dicho escri- 
to se señalan los motivos de la solicitud de renuncia, entre las cuales se encuentra la "[...] imposibilidad de lograr un acuerdo en torno al texto de los futuros estatutos de la universidad, en cuanto las observaciones planteadas de manera reiterada por este Ministerio no fueron adecuadamente atendidas" (Chile, 2016). En particular, la disputa se centró en la estructura del gobierno universitario, es decir, entre la búsqueda de eficiencia o participación. De tal modo, el Ministerio realizó un conjunto de modificaciones a la propuesta con el objetivo de darle más eficiencia a la puesta en marcha de la universidad, posponiendo la constitución de aquellos órganos de gobierno comprometidos con la participación de la comunidad local (Cáceres, 2011; Rivera-Polo et al., 2018).

Al respecto, cabe señalar que la primera propuesta de estatutos de la Universidad - redactados de forma participativa, tal como exigía la ley, para lo que se reactivó a la antigua Comisión Regional, aun cuando esta ya había cesado sus funciones - contemplaba un Consejo Social con mayores atribuciones que el actual. Así, se transitó desde un CS orientado a facilitar y contribuir el encuentro entre la Universidad y la comunidad regional (Universidad de Aysén, 2016c) a otro cuya finalidad es "[...] mantener informado al Rector o la Rectora de la Universidad en relación con las necesidades y problemáticas efectivas de la región” (Universidad de Aysén, 2018b, p. 1) ${ }^{8}$. Si bien la intervención del gobierno central respecto de la función del CS no fue menor, en tanto que redujo su capacidad de acción, no logró eliminar este espacio único de participación. Así, la existencia del CS abre la posibilidad de repensar el sentido territorial de la Universidad. En la siguiente sección reflexionamos sobre las posibilidades que abre el CS.

\section{La posibilidad: el Consejo Social de la Universidad de Aysén}

El análisis anterior muestra que la Universidad de Aysén, aunque tiene que cumplir con las obligaciones que derivan de su condición de universidad estatal, contiene una posibilidad, la de convertirse en algo distinto, de pensarse de manera distinta. Esta posibilidad descansa, como hemos sostenido, en la existencia del CS. Sim embargo, la existencia del CS también produce tensiones y desafíos que requieren un mayor análisis.

A partir de las entrevistas conducidas con integrantes del CS y ex autoridades, hemos identificado tres tipos de tensiones y desafíos que emergen como consecuencia de la existencia del CS. En primer lugar, tensiones que provienen del diseño del CS, es decir, tensiones que podrían ser denominadas internas. En segundo lugar, las demandas que nacen del territorio y que tensionan al CS. Y, en tercer lugar, las tensiones que derivan del hacer del CS y que impactan e interpelan al proyecto universitario. La siguiente tabla resume las tensiones identificadas. 
Tabla 2 - Principales tensiones y desafíos que derivan de la existencia del Consejo Social

\begin{tabular}{|c|c|}
\hline Direccionalidad & Principales tensiones y desafíos \\
\hline $\begin{array}{l}\text { 1. Tensiones } \\
\text { internas del CS }\end{array}$ & $\begin{array}{l}\text { - Las atribuciones limitadas debido al carácter consultivo del } \\
\text { CS } \\
\text { - El tamaño para asegurar representatividad versus operati- } \\
\text { vidad } \\
\text { - El peligro de captura por ciertos integrantes (desequilibrio } \\
\text { de influencia) } \\
\text { - La excesiva presencia de representantes de la Universidad en } \\
\text { el CS } \\
\text { - El riesgo de anular el aporte del CS por influencia excesiva de } \\
\text { las autoridades internas }\end{array}$ \\
\hline $\begin{array}{l}\text { 2. Tensiones } \\
\text { desde el territo- } \\
\text { rio al CS }\end{array}$ & $\begin{array}{l}\text { - El carácter consultivo del CS no permite un vínculo sustan- } \\
\text { tivo } \\
\text { - La escasa prioridad y respuesta oportuna de la universidad a } \\
\text { los problemas territoriales } \\
\text { - Representación incompleta de la sociedad y del territorio } \\
\text { - El riesgo de que integrantes del CS no representen al organis- } \\
\text { mo o sector del que provienen, y que respondan a intereses } \\
\text { personales } \\
\text { - Los cambios abruptos de integrantes que representan al } \\
\text { sector público debido al cambio de signo de gobierno central } \\
\text { y regional } \\
\text { - El CS también puede prevenir conflictos de interés, canali- } \\
\text { zándolos de forma transparente si se explicitan }\end{array}$ \\
\hline $\begin{array}{l}\text { 3. Tensiones } \\
\text { desde el CS } \\
\text { hacia la univer- } \\
\text { sidad }\end{array}$ & $\begin{array}{l}\text { - La tensión entre autonomía universitaria versus vinculación } \\
\text { con el medio } \\
\text { - La tensión entre la producción de conocimiento científico } \\
\text { como respuesta al modelo prevalente y reconocimiento de } \\
\text { otros saberes que emergen desde territorio } \\
\text { - La tensión entre un perfil de académico/a desconectado de } \\
\text { las problemáticas del territorio y las necesidades de la comu- } \\
\text { nidad local }\end{array}$ \\
\hline
\end{tabular}

Fuente: Elaboración propia a partir de análisis documental y entrevistas a integrantes del Consejo Social de la Universidad de Aysén.

Reconociendo que todas estas tensiones merecen un tratamiento especial, nos interesa concentrarnos, más bien, en el tercer punto de la tabla, es decir, en las tensiones que irrumpen desde el CS hacia la universidad. Tres temas (tensiones) emergieron del análisis de las entrevistas. En primer lugar, la tensión entre autonomía universitaria y vinculación territorial, es decir, en qué medida el principio de autonomía que define al proyecto moderno de universidad queda suspendido o cuestionado al momento de encauzar el proyecto territorial. En segundo lugar, la tensión entre conocimiento científico y conocimientos "artesanales", es decir, hasta qué punto la producción de conocimiento bajo la agenda neoliberal y el proyecto moderno son modificados por nuevas fuentes de conocimiento. En tercer lugar, el rol del estamento académico en el proceso de reordenamiento y reconfiguración de saberes científicos antes mencionado.

Autonomía universitaria y vinculación territorial - "El territorio es el protagonista de las soluciones" (Autoridad Universitaria). Esta afir- 
mación indica los esfuerzos que realiza la Universidad de Aysén para definir y establecer una vinculación permanente con su territorio. Esta voluntad ha sido potenciada y, a la vez, resignificada por la existencia del CS, más allá de las exigencias de las políticas nacionales de vinculación con el medio. Uno de los integrantes del Consejo, representante de la sociedad civil, lo señala de la siguiente manera:

[...] lo que falta es la transversalización de la cultura en todas las carreras [...] las carreras adolecen del espíritu de Aysén (Integrante del Consejo Social).

Así, no solo se trata de la Universidad en el territorio, sino también el territorio en la universidad, es decir, lo que recientemente se ha denominado vinculación bidireccional en la acreditación institucional. No obstante, lo que redefine e impugna el CS es el alcance y nivel de dicha vinculación, la que se considera meramente instrumental. A partir de ello, la Universidad ha propuesto un sistema de vinculación que permite mayor presencia de la universidad en el territorio. Un ejemplo de aquello es la forma en que un problema de investigación es definido. Así lo señala un profesional de la Universidad:

[...] las necesidades de la región se levantan a través del trabajo de los Consejeros desplegados en los distintos territorios de la región (Profesional de la Universidad).

Un representante del estamento académico plantea dicha necesidad de la siguiente forma:

[...] las necesidades de la región han sido postergadas por muchos años, entonces yo de manera personal no tengo cara para decir que yo voy a seguir trabajando con los tomates de Angol [otra región del país] o con los duraznos de Talca [otra región] porque aquí hay una demanda de otras necesidades [...] y ahí nacen los proyectos de investigación (Académico de la universidad).

Lo mismo ocurre a nivel de docencia, donde se observa que la universidad ha definido las carreras y sus planes de estudio atendiendo las expectativas de la comunidad local. De esta forma, la universidad ha avanzado hacia un mayor vínculo incorporando

[...] en los planes de estudio un apartado que indica cuáles van a ser las actividades de vinculación que ellos [los académicos y académicas] van a realizar en docencia (Integrante del Consejo Social).

Todo lo anterior muestra un esfuerzo por definir y desplegar la vinculación territorial en todas las funciones de la universidad, por ejemplo, a través de carreras vinculadas con los sectores productivos. Sin embargo, esto no es lo mismo que "transversalizar la cultura de Aysén en todas las carreras”, como fue planteado por el representante civil en el CS.

[...] las carreras que se ofrecen no son pertinentes [...] ¿por qué otra vez esa carrera? [...] La gente tenía la expectativa que se desarrollara en esa línea [mayor pertinencia con el territorio] y las carreras fueron muy tradi- 
cionales [...] lo mismo con los planes de estudio, no contemplan las particularidades de la región de Aysén (Integrante del Consejo Social).

Aquí se observa la tensión entre una vinculación instrumentalizada - debido a las presiones que impone la acreditación institucional - y una vinculación territorializada, como lo entienden algunos representantes del CS, dadas las características de la región donde se ubica la universidad.

Por otra parte, una vinculación entendida como renuncia a la autonomía o libertad académica en pos de una mayor vinculación territorial se contrapone con la visión que algunos académicos sostienen, particularmente en la producción de conocimiento. Por una parte, un académico señala que

[...] si bien la vinculación con el medio es muy importante, especialmente en esta región debido al aislamiento, lo nueva que es la universidad, el hecho de realizar investigación con la región es más importante que en otros lados [...] los que estamos realizando investigación básica, es decir, que estamos explorando o trabajando con pacientes, necesitamos otro tipo de financiamiento [...] porque es distinto el experimento que uno realiza y eso no se premia [...] (Integrante del cuerpo académico de la universidad).

\section{Por otra parte,}

[...] el hecho de que la universidad sea nueva permite mayor libertad para elegir los temas de investigación, aunque siempre el tema de las necesidades regionales está presente (Integrante del cuerpo académico de la universidad).

En esa misma línea, se insiste en la libertad académica como condición para la generación de conocimiento.

Una de las herramientas más importantes es la libertad de pensamiento, eso es lo más importante [...] (Integrante del cuerpo académico de la universidad).

Esta tensión ha sido particularmente profunda puesto que la capacidad de territorializar a la universidad depende de la suspensión de dicha forma de autonomía y libertad académica. Ya no se trata de las necesidades del Estado central o del mercado, sino de las necesidades del territorio, de la comunidad local. En definitiva, el CS ha reforzado la necesidad de suspender el concepto de autonomía de la universidad moderna. La impugnación del CS se hace evidente cuando se reflexiona sobre el contenido de la vinculación bidireccional. No solo se trata de desplegar a la universidad en el territorio, sino que el territorio se despliegue en las funciones y prácticas de la universidad.

Conocimiento científico versus conocimientos artesanales - En el contexto de la actual forma de generar conocimiento, es decir, a través de fondos concursables, algunos representantes del cuerpo académico de la universidad señalan que lo relevante es la internalización de ciertas estrategias epistémicas y metodológicas para responder a dichas exigencias. 
[...] ese conocimiento forzado [aplicar a proyectos concursables], permite que uno se pueda ganar un proyecto en los próximos años [...] ese análisis que uno hace en el doctorado, en cuatro o cinco años, muchos de mis colegas lo están haciendo de manera muy apresurada, e incorporándolo casi a su orgánica, desde cuestionarse el objetivo desde el punto de vista de la aplicabilidad hasta la lectura que puede tener el revisor del proyecto, que eso a uno no se lo enseñan en el doctorado [...] (Integrante del cuerpo académico de la universidad).

En ese sentido, se destaca además la necesidad de aplicar el método científico, es decir, el despliegue de prácticas científicas que permitan darle forma a un proyecto de investigación.

En el caso mío, el factor principal [para postular a un proyecto] viene de la vieja escuela, mi profesora tutora se sentaba al lado mío, [...] y me explicaba que uno no puede postular a un proyecto sin antes poner la idea en juego [...] anda al laboratorio primero, observa, pero observa, no mires, porque nosotros miramos a diario, observa que está pasando, y ten tus datos preliminares y luego apunta al proyecto [...] (Integrante del cuerpo académico de la universidad).

Sin embargo, algunas voces del CS advierten la necesidad de reconocer otros tipos de saberes, prácticas, virtudes y experiencias, más allá de los que provienen del conocimiento científico, enraizados en la cultura de la región y que aún perduran a través de múltiples prácticas. Como indica un integrante del CS, esto se debe principalmente a la historia de la región, porque

Aysén desde la colonización fue siempre autovalente respecto al Estado, se hacían cosas y luego se pedía permiso al Estado. Siempre estuvo la gente antes del Estado. La gente luego trata de validar lo que ha hecho con el Estado. Lo mismo con la Universidad. La gente ya sabía cómo quería la universidad (Integrante del Consejo Social).

\section{Por lo tanto,}

[...] en la región de Aysén hay conocimientos/saberes de la medicina no tradicional que todavía están vigentes y que se pueden integrar (Integrante del Consejo Social).

Así, se releva la necesidad de aplicar otro tipo de miradas, de observación, de prácticas, es decir,

Volver a mirar la historia natural de Aysén [...] Una generación atrás se pegaban los huesos con hueseras, se atendían los partos en el campo.... hay un remanente de medicina no tradicional que todavía están vigentes y que se puede incorporar (Integrante del Consejo Social).

Esta perspectiva ha quedado plasmada en las actas del CS de la universidad, donde se ha insistido en la necesidad de incorporar la historia, cultura y espíritu de Aysén en distintos ámbitos de la universidad. Así, por ejemplo, en el acta de la primera sesión se indica que "El Consejo Social puede proponer a la Universidad coloque temas con contenido intelectual, independencia política, y ayudar a pensar la región" (Universidad de Aysén, 2018a, p. 4). En la tercera sesión se vuelve a insistir 
que en la necesidad de "rescatar las tradiciones y costumbres ancestrales" (Universidad de Aysén, 2019, p. 3) que perviven en el territorio.

Este requerimiento no supone abandonar el conocimiento producido a través del método científico, sino más bien abrirse a esos otros saberes, a reconocerlos en su propio mérito. Esto es lo que Santos (2018) destaca como "conocimientos artesanales", es decir, aquellos saberes que provienen de diversas culturas que se traducen a su vez en una variedad de prácticas pero que tienen algo en común: son saberes producidos estrechamente con la comunidad.

Esto es también lo que estuvo a la base de las propuestas de acceso de la Universidad, como se discutió más arriba. Hay un cuestionamiento desde el territorio sobre la capacidad de la universidad no solo de reconocer saberes sino también otras experiencias de vida, muy distintas a las del resto de las regiones del país. Esto queda claramente establecido en las palabras de una ex autoridad de la universidad.

[...] los y las jóvenes que viven en esta región extrema de Aysén tienen experiencias de vida, oportunidades escolares y formativas diferentes al resto del país, porque la aptitud para el estudio, la inteligencia, la voluntad y el esfuerzo están repartidos de forma homogénea en la sociedad, pero el conocimiento, así como el mero entrenamiento para contestar la prueba de acceso no lo está y eso ha generado brechas que son insalvables e injustas (ex autoridad universitaria).

A partir de lo anterior, es necesario preguntar en qué medida estos saberes y experiencias son reconocidos por la universidad. Según Santos (2018), la capacidad de que estos saberes perduren "depende de la eficacia de tales conocimientos en fortalecer las luchas concretas y las resistencias contra la opresión, es decir, el modo en el cual tales conocimientos contribuyen a maximizar las posibilidades de éxito de dichas luchas y resistencias" (Santos, 2018, p. 43). Esta definición, si bien es loable y merece un debate más amplio, no incluye aquellos saberes y experiencias que provienen de prácticas culturales no orientadas a la lucha social, sino al bienestar de las personas y el territorio. Por ejemplo, en Aysén hay luchas sociales orientadas a proteger la privatización del acceso a lagos y contrarrestar la explotación minera. Más allá de estas distinciones, lo que este tipo de conocimiento pone en tensión son las virtudes epistémicas, tales como la objetividad y neutralidad, que están a la base del conocimiento científico (Agazzi, 2014; Reiss; Sprenger, 2014). Y ese es precisamente el impacto que produce el CS en la universidad, es decir, revela los límites de estos conceptos y prácticas.

El rol de la academia: productividad versus compromiso territorial - Es posible observar una tensión entre la búsqueda de rendimiento individual y compromiso territorial en la academia. La producción de conocimiento requiere del cumplimiento de ciertos niveles de productividad, los que son evaluados por los procesos de acreditación institucional y las propias comunidades disciplinares. Esta situación es descrita de la siguiente forma: 
La ciencia se mecaniza mucho, es producir, producir, producir [...] (Integrante del cuerpo académico de la universidad).

Es interesante observar cómo el sistema de acreditación define ciertas prácticas académicas, como se describe a continuación.

[...] constantemente estoy pensando en lo que se propone para investigar y lo que es acreditable [proceso de acreditación institucional]...yo lo tengo incorporado [...] si esto es acreditable, entonces hagámoslo [...] (Integrante del cuerpo académico de la universidad).

Así, por ejemplo, algunos integrantes del cuerpo académico utilizan estrategias para responder a las obligaciones que derivan de la productividad.

[...] necesitamos tener la continuidad de un Fondecyt, entonces a mí se me protege los últimos seis meses para generar una idea...porque en el laboratorio yo soy la única que tiene doctorado [...] A mis colegas le ayudamos a su formación de desarrollo curricular, de esta manera, cuando postulen a su doctorado ya tienen líneas de investigación vigente, proyectos postulables, y trabajan su productividad científica. Nosotros en el laboratorio hemos creado esta red [...] pensando siempre en la acreditación (Integrante del cuerpo académico de la universidad).

La impugnación que emerge del CS, al menos como posibilidad, es precisamente la de suspender el saber, o más precisamente, redefinir la relación entre saber e individualidad puesto que

[...] Uno de los problemas es la soberbia del saber [...] (Integrante Consejo Social).

O, como lo señala Santos, promover la capacidad de los académicos y académicas "de reaprender, de recordar de otro modo" (Santos, 2018, p. 154). Un integrante del CS lo indica de la siguiente manera:

[...] los grados académicos no son garantía de que [los académicos y académicas] tengan la disposición de abrirse a otros temas más allá de sus líneas de investigación; hay que volver a reaprender. Aquí [en Aysén] todo es diferente, es una región que era desconocida para el país [...] Culturalmente es muy distinto (Integrante Consejo Social).

En este sentido, lo que releva esta postura es la necesidad de un cuerpo académico que territorialice su conocimiento y experiencia. No en el sentido de desplegar o comunicar el saber producido, que es algo más bien unidireccional, sino de vivir la bidireccionalidad. Esto es lo que Santos considera como un aspecto fundamental de la subversidad y que conduce a repensar las prácticas pedagógicas desde una nueva actitud, la de desaprender para reaprender. Las palabras de un representante del cuerpo académico de la universidad son claras al respecto:

¿Esta maquinaria de producción científica siempre responde a las necesidades del ser humano? En el contexto del Covid esto es un análisis que la ciencia se está haciendo hoy en día. Nos hemos dado cuenta que en Chile la investigación es de punta [...] pero nos hace falta un poco más lo humano y no solo responder al mundo científico de manera cuadrada (Integrante del cuerpo académico de la universidad). 
Aquí la palabra humano parece indicar una consideración que va más allá de la productividad científica. Aquí nuevamente es útil recordar lo que señala Santos:

[...] los [académicos y académicas] deben esforzarse en pensar en ellos mismos sin títulos, certificados o diplomas, y sentir el aura de la universidad como una carga más que una ventaja, y reaprender a distinguir la autoridad del conocimiento de la autoridad de la institución que lo reproduce (Santos, 2018, p. 284).

El aura, que muchas veces se pierde en la reproducción infinita del saber, como bien lo advirtió Benjamin (2005), aquí, al parecer, emerge y se reproduce en el territorio.

\section{Algunas conclusiones: el devenir subversivo de la universidad territorial}

En este artículo hemos tratado de reflexionar críticamente sobre el rol de la Universidad Estatal de Aysén y, a la vez, pensar la posibilidad de una subversidad en dicho territorio. En particular, nos ha interesado analizar cómo la universidad ha respondido simultáneamente a las normativas, políticas nacionales y expectativas de la comunidad local, así como también qué tipo de tensiones produce la existencia del CS. Hemos intentado señalar algunas tensiones que emergen de dicha relación y cómo ellas desafían el proyecto territorial de la universidad.

El sentido territorial de la universidad indica que ella piensa y constituye al territorio. A diferencia de la lógica mercantil, que carece de referencialidad universal o que las reactiva de tal manera que pierden su sentido social y construcción de lo común, la universidad territorial aboga por la construcción de una época, que en este caso es la constitución de un territorio. Sin embargo, dicha constitución no implica una vuelta a los principios normativos, o a ciertas referencias universales, del proyecto modernizador y neoliberal, sino más bien a la radicalización de la localidad y la temporalidad en que ocurre dicha constitución. Por tal razón, la palabra territorio (lugar y tiempo) adquiere una connotación doble: es impugnadora y, a la vez, constituyente. De esta manera, la universidad territorial no es simplemente un buzón de problemas y necesidades sociales a los cuales debe responder oportunamente, como promueven las políticas nacionales. Su misión es distribuirse en el territorio de tal manera que llegue a ser territorio ella misma.

En ese sentido, los actores de la universidad plantean que el CS tiene el potencial de convertirse en el espacio de la impugnación, de la imaginación, de nuevas formas de hacer universidad. Así, nos parece relevante concebir al CS como la esfera pública que permite concretar el proyecto territorial de la universidad. Es el lugar donde se reúnen las distintas voces de la comunidad local con el propósito de discutir las necesidades de la región. Sin embargo, este espacio no debiera concebirse como una esfera pública que busca acuerdos en función del des- 
pliegue de una racionalidad comunicativa (Barnett, 2008). Al contrario, se trata de un espacio que es necesario pensarlo desde la conflictividad, es decir, un lugar que es a la vez opaco, jerárquico y fragmentado. En otras palabras, un lugar participativo y deliberativo, de acuerdos y desacuerdos, que descansa sobre relaciones de poder desiguales y que, por tal motivo, encarna la conflictividad de la vida social (Komporozos-Athanasiou et al., 2019). Estos supuestos no son simples de traducir puesto que lo que ha producido la agenda neoliberal, o mejor, lo que esta agenda le ha quitado a las universidades es precisamente su capacidad para pensar. Las prácticas homogeneizadoras de la acreditación (Morley, 2003) - por ejemplo, la idea de vinculación con el medio - han suspendido la capacidad deliberativa de las universidades.

Frente a ello, el CS corre varios riesgos que deben ser considerados cuidadosamente. Uno de ellos, como mencionamos, es que quede subsumido bajos las lógicas de la acreditación. Otro riesgo es más bien interno. Dado que el CS navega entre lo externo (el territorio) y lo interno (la propia universidad), corre el riesgo de ser visto como una entidad externa al interior de la universidad. Así, parece relevante repensar el carácter vinculante y consultivo del CS en tanto que la forma en que ella misma sea territorio (la universidad) supone una articulación más sustantiva entre el CS y la universidad.

Teniendo presente estas consideraciones, es posible concluir que la Universidad de Aysén, junto con el Consejo Social, irrumpe para impugnar y dislocar al mercado en educación superior, pero además, a las premisas de la universidad moderna (por ejemplo, autonomía y objetividad). Lo que muestra la instalación de la universidad es que no es posible sostener una Universidad de estas características, territorial y fiel al mandato social, bajo los mecanismos de mercado, y menos aún con la intervención del gobierno central o regional. Eso es lo que muestra el ejercicio en un territorio extremo como el de Aysén, y cuyos resultados son extensivos a todo territorio, puesto que esos mismos problemas e impedimentos que se encuentran allí concentrados, aparecen ubicuos de forma difusa en otros lugares. El proyecto territorial solo es posible bajo una esfera de lo público que es necesario auscultar y analizar en profundidad. Esa reflexión es fundamental en el sistema de educación superior chileno, donde es necesario comenzar con un proceso profundo de desmercantilización. La experiencia de la Universidad de Aysén, en el marco analítico que hemos propuesto, puede ser un ejemplo relevante para pensar e imaginar.

Recibido el 1 de junio de 2021 Aprobado el 9 de agosto de 2021

\section{Notas}

1 "Movimiento Social de Aysén: Tu problema es mi problema".

2 Por ejemplo, la primera universidad popular creada en el norte de África fue en Alexandria, Egipto, bajo la iniciativa de trabajadores anarquistas italianos y griegos. En América Latina, la primera universidad de este tipo fue creada en 1921 en Perú, la Universidad Popular Gonzáles Prada (Santos, 2018). 
3 Esto se evidencia con claridad en la carta entregada por el Ministerio de Educación a la Rectora de la universidad, en donde se esgrimen los principales motivos para solicitar la renuncia (agosto 2016).

4 Se dejó impedido por ley el avance de la gratuidad más allá del séptimo decil, dejando un amarre al crecimiento del PIB y se mantuvo el otorgamiento del beneficio de forma individual, con forma de financiamiento a la demanda en vez de financiamiento a las instituciones.

5 La propuesta no alcanzó a ser presentada al Ministerio de Educación. Los autores tuvieron acceso a los informes internos elaborados para sustentar la propuesta.

6 Esta Prueba está siendo transformada desde la evaluación de contenidos curriculares de la enseñanza media hacia la evaluación de competencias fundamentales para estudiar en la educación superior. En 2020 se aplicó por primera vez la Prueba de Transición.

7 Es relevante señalar, que más allá de las tensiones, resistencias y el rechazo a esa propuesta de inclusión realizada en el año 2016, recientemente en el año 2019 se introdujeron elementos semejantes para favorecer la inclusión en las regiones denominadas extremas.

8 Es importante señalar que, en virtud de la discusión interna en el CS y entre las autoridades de la Universidad, el último reglamento de funcionamiento del CS incluye "[...] ser una instancia propositiva que permita reconocer las necesidades y problemáticas de la región, potenciando la relación de la universidad con su entorno local en los ámbitos estratégicos definidos por la universidad [...] y mantener una estrecha relación entre la comunidad regional y la Universidad de Aysén" (Universidad de Aysén, 2018b, p. 2).

\section{Referencias}

AGAZZI, Evandro. Scientific objectivity and its contexts. Heidelberg; New York; Dordrecht; London: Springer, 2014.

BALL, Stephen J.; MAGUIRE, Meg; BRAUN, Annette. How schools do policy: policy enactments in secondary schools. Oxfordshire: Routledge, 2012.

BALL, Stephen J.; OLMEDO, Antonio. Care of the self, resistance and subjectivity under neoliberal governmentalities. Critical Studies in Education, v. 54, n. 1, p. 85-96, 2013. https://doi.org/10.1080/17508487.2013.740678.

BARNETT, Clive. Convening Publics: The parasitical spaces of public action. In: COX, Kevin; LOW, Murray; ROBINSON, Jennifer (Ed.). The SAGE Handbook of Political Geography. Thousand Oaks: Sage Publications Ltd. 2008. P. 403-417. http://www.sagepub.co.uk/booksProdDesc.nav?prodId=Book225561.

BASSEY, Michael. Case study research in educational settings. Buckingham: Open University Press, 1999.

BENJAMIN, Walter. Libro de los pasajes. Madrid: Akal Ed., 2005.

BERNASCONI, Andrés. Is There a Latin American Model of the University? Comparative Education Review, v. 52, n. 1, p. 27-52, 2008. https://doi. org/10.1086/524305.

BRUNNER, José Joaquin (Ed.). El conflicto de las universidades: entre lo público y lo privado. Santiago: Ediciones Universidad Diego Portales, 2011. 
CÁCERES, Eugenio. El financiamiento de la educación superior en la década de los ochenta. In: JIMÉNEZ DE LA JARA Mónica; DURÁN DEL FIERRO, Francisco (Ed.). Un recorrido por la historia reciente de la educación superior chilena: 1967-2011. Santiago: Santo Tomás; Aequalis Foro de Educación Superior, 2011. P. 97-119.

CHILE. Ministerio de Educación. Ley 20842. Crea las Universidades Estatales de la Región de O’Higgins y de la Región de Aysén. Santiago, 2015. Disponible en: https://www.bcn.cl/leychile/navegar?idNorma=1080237. Acceso en: 02 jun. 2021.

CHILE. Ministerio de Educación. Solicitud de renuncia a la Rectora de la Universidad de Aysén [Personal communication]. Santiago, 2016.

COMISIÓN EJECUTIVA REGIONAL. Sentando las bases para habitar de otro modo el planeta. Propuesta de la Comisión Ejecutiva para crear la Universidad Estatal de Aysén. Coyhaique: Ministerio de Educación, 2015.

DELEUZE, Gilles; GUATTARI, Félix. Anti-Oedipus: capitalism and schizophrenia. Minneapolis: University of Minnesota Press, 1983.

DURÁN DEL FIERRO, Francisco. Educación superior y desarrollo territorial: la Universidad de Aysén en el diagrama neoliberal. Magíster En Gestión y Políticas Públicas (MGPP), Santiago de Chile, Universidad de Chile, Estudios de Caso, n. 137, 2016. Disponible en: http://www.sistemaspublicos.cl/wp-content/ uploads/2017/04/137Francisco-Duran-2.pdf. Acceso en: 02 jun. 2021.

JOFFE, Helene. Thematic Analysis. In: HARPER, David; THOMPSON, Andrew R. (Ed.). Qualitative Research Methods in Mental Health and Psychotherapy. Hoboken: John Wiley \& Sons, 2011. P. 209-223. Disponible en: https://doi. org/10.1002/9781119973249.ch15. Acceso en: 02 jun. 2021.

KOMPOROZOS-ATHANASIOU, Aris; RENEDO, Alicia; MCKEVITT, Christopher. Citizen Participation in Neoliberal Times. Sociological Research Online, v. 24, n. 3, p. 370-375, 2019. Disponible en: https://doi.org/10.1177/1360780419835562. Acceso en: 02 jun. 2021.

LEMAITRE, María José (Ed.). Diversidad, autonomía, calidad. Desafíos para una educación superior para el siglo XXI. Santiago de Chile: Centro Interuniversitario de Desarrollo); RIL Editores, 2019. Disponible en: https://cinda.cl/publicacion/diversidad-autonomia-calidad-desafios-para-una-educacion-superiorpara-el-siglo-xxi/. Acceso en: 02 jun. 2021.

LEMAITRE, María José; DURÁN DEL FIERRO, Francisco. Hacia una nueva arquitectura del sistema de educación superior: el régimen de lo público. Santiago de Chile: Aequalis, 2013.

MARGINSON, Simon. Higher Education and Public Good: Higher Education and Public Good. Higher Education Quarterly, Hoboken, v. 65, n. 4, p. 411-433, 2011. Disponible en: https://doi.org/10.1111/j.1468-2273.2011.00496.x. Acceso en: 02 jun. 2021.

MORLEY, Louise. Quality And Power In Higher Education. New York: McGrawHill Education, 2003. Disponible en: http://ebookcentral.proquest.com/lib/ ucl/detail.action?docID=292128. Acceso en: 02 jun. 2021.

MUSSELIN, Christine. New forms of competition in higher education. Socio-Economic Review, v. 16, n. 3, p. 657-683, 2018. Disponible en: https://doi. org/10.1093/ser/mwy033. Acceso en: 02 jun. 2021.

ORELLANA, Victor et al. Entre el mercado gratuito y la educación pública. Santiago: LOM Ediciones, 2018. 
Territorialidad y Neoliberalismo

OSSA, Carlos. El ego explotado. Capitalismo cognitivo y precarización de la Creatividad. Santiago: Ediciones Departamento de Artes Visuales/Facultad de Artes, Universidad de Chile, 2016.

PÉREZ, Miguel. Movimiento social de Aysén. Un caso de análisis de incidencia ciudadana en la agenda de políticas públicas. Revista Estudios de Políticas Públicas, v, 1, n. 1, p. 100-116, 2015.

PEY, Roxana. Una reflexión sobre la internacionalización de la educación superior y los desafíos para las universidades chilenas. Anales Del Instituto de Chile, Santiago, v. XXV, p. 75-86, 2006.

PEY, Roxana. Gratuita y estatal...extrema. In: THAYER Willy; COLLINGWOODSELBY, Elizabeth; SERRANO Mary Luz; FREIRE RODRÍGUEZ, Raúl (Ed.). La Universidad (im)posible. Santiago: Ediciones Macul; Universidad Metropolitana de Ciencias de la Educación, 2018.

REISS, Julian; SPRENGER, Jan. Scientific objectivity. In: ZALTA, Edward (Ed.). Stanford Encyclopedia of Philosophy. Stanford: Metaphysics Research Lab; Stanford University, 2014

RIVERA-POLO, Felipe; RIVERA-VARGAS, Pablo; ALONSO-CANO, Cristina. Ni nacional, ni empresarial:legitimidad territorial universitaria. El caso de la Universidad de Aysén en Chile. Revista Izquierdas, p. 2321-2350, 2020.

RIVERA-POLO, Felipe; RIVERA-VARGAS, Pablo; ALONSO-CANO, Cristina. Una mirada territorial al sistema universitario chileno. El caso de la Universidad regional de Aysén (UAY). Estudios Pedagógicos, Valdivia, v. 44, n. 1, p. 427-443, 2018. Disponible en: https://doi.org/10.4067/S0718-07052018000100427. Acceso en: 02 jun. 2021.

ROCK, Juan Antonio et al. Vinculación del desarrollo regional y la educación superior en Chile: diagnóstico y propuestas. Santiago: Aequalis, 2013.

SALAZAR ZEGERS, Jose Miguel; LEIHY, Peodair Seamus. El Manual Invisible: Tres décadas de políticas de educación superior en Chile (1980-2010). Education Policy Analysis Archives, v. 21, n. 34, 2013. Disponible en: https://doi. org/10.14507/epaa.v21n34.2013. Acceso en: 02 jun. 2021.

SANTOS, Boaventura de Sousa. La universidad popular del siglo XXI. Fondo Editorial de la Facultad de Ciencias Sociales, UNMSM, 2006. Disponible en: http://books.google.com/books?id=DuycAAAAMAAJ. Acceso en: 02 jun. 2021.

SANTOS, Boaventura de Sousa. The end of the cognitive empire: the coming of age of epistemologies of the South. Durham: Duke University Press, 2018.

SIES. Informe de Matrícula en la Educación Superior en Chile - 2020. Santiago: Ministerio de Educación, 2020. Disponible en: https://www.mifuturo.cl/ wp-content/uploads/2020/07/Informe-matricula_2020_SIES.pdf. Acceso en: 02 jun. 2021.

THAYER, Willy et al. (Ed.). La Universidad (im)posible. Santiago: Ediciones Macul; Universidad Metropolitana de Ciencias de la Educación, 2018.

UNIVERSIDAD DE AYSÉN. Acta Consejo Social Provisorio. Segunda Sesión Ordinaria. Coyhaique, 2016a.

UNIVERSIDAD DE AYSÉN. Acta Consejo Social Provisorio. Tercera Sesión Ordinaria. Coyhaique, 2016b.

UNIVERSIDAD DE AYSÉN. Propuesta de Estatuto de la Universidad de Aysén. Coyhaique, 2016c. 
UNIVERSIDAD DE AYSÉN. Acta Consejo Social. Primera Sesión Ordinaria. Coyhaique, 2018a.

UNIVERSIDAD DE AYSÉN. Confórmese el Consejo Social de la Universidad de Aysén según lo dispuesto en el Artículo 14 del DFL $\mathrm{N}^{\circ} 7$ y fija normas básicas para su funcionamiento. Coyhaique, 2018b.

UNIVERSIDAD DE AYSÉN. Acta Consejo Social. Tercera Sesión Ordinaria. Coyhaique, 2019.

VAN VUGHT, Frans. Diversity and Differentiation in Higher Education. In: VAN VUGHT, Frans (Ed.). Mapping the Higher Education Landscape. V. 28. Netherlands: Springer Netherlands, 2009. P. 1-16. Disponible en: https://doi. org/10.1007/978-90-481-2249-3_1. Acceso en: 02 jun. 2021.

VILLALOBOS-RUMINOTT, Sergio. Equivalencia neoliberal e interrupción nómica: el conflicto de las facultades como contrato social. In: THAYER, Willy et al. (Ed.). La Universidad (im)posible. Santiago: Ediciones Macul; Universidad Metropolitana de Ciencias de la Educación, 2018.

YIN, Robert K. Case study research: design and methods. 3. ed. Thousand Oaks: Sage Publications, 2003.

Francisco Durán del Fierro es sociólogo y actualmente realiza sus estudios de doctorado en Inglaterra; su proyecto de investigación explora la dimensión ética de la crítica en la academia en Chile. Ha sido profesor e investigador en la Universidad de Chile.

ORCID: https://orcid.org/0000-0003-0534-5342

E-mail: f.duran@ucl.ac.uk

Roxana Pey Tumanoff es Licenciada en Biología y Doctora en Ciencias con mención en Biología Celular de la Universidad de Chile. Realizó una estadía postdoctoral en Alemania y diversas estadías de investigación científica y sobre educación superior en Estados Unidos, Alemania y Holanda.

ORCID: https://orcid.org/0000-0002-2143-8242

E-mail: roxana.pey@gmail.com

Editor a cargo: Luís Armando Gandin

Este é um artigo de acesso aberto distribuído sob os termos de uma Licença Creative Commons Atribuição 4.0 Internacional. Disponível em: <http:// creativecommons.org/licenses/by/4.0>. 